PROCEEDINGS OF THE

AMERICAN MATHEMATICAL SOCIETY

Volume 138, Number 2, February 2010, Pages 545-551

S 0002-9939(09)10119-3

Article electronically published on September 29, 2009

\title{
A NOTE ON THE BUCHSBAUM-RIM MULTIPLICITY OF A PARAMETER MODULE
}

\author{
FUTOSHI HAYASAKA AND EERO HYRY
}

(Communicated by Bernd Ulrich)

\begin{abstract}
In this article we prove that the Buchsbaum-Rim multiplicity $e(F / N)$ of a parameter module $N$ in a free module $F=A^{r}$ is bounded above by the colength $\ell_{A}(F / N)$. Moreover, we prove that once the equality $\ell_{A}(F / N)=e(F / N)$ holds true for some parameter module $N$ in $F$, then the base $\operatorname{ring} A$ is Cohen-Macaulay.
\end{abstract}

\section{INTRODUCTION}

Let $(A, \mathfrak{m})$ be a Noetherian local ring with the maximal ideal $\mathfrak{m}$ and $d=\operatorname{dim} A>$ 0 . Let $F=A^{r}$ be a free module of rank $r>0$, and let $M$ be a submodule of $F$ such that $F / M$ has finite length and $M \subseteq \mathfrak{m} F$.

In their article [5] from 1964 Buchsbaum and Rim introduced and studied a multiplicity associated to a submodule of finite colength in a free module. This multiplicity, which generalizes the notion of Hilbert-Samuel multiplicity for ideals, is nowadays called the Buchsbaum-Rim multiplicity. In more detail, it first turns out that the function

$$
\lambda(n):=\ell_{A}\left(\mathcal{S}_{n}(F) / \mathcal{R}_{n}(M)\right)
$$

is eventually a polynomial of degree $d+r-1$, where $\mathcal{S}_{A}(F)=\bigoplus_{n \geq 0} \mathcal{S}_{n}(F)$ is the symmetric algebra of $F$ and $\mathcal{R}(M)=\bigoplus_{n>0} \mathcal{R}_{n}(M)$ is the image of the natural homomorphism from $\mathcal{S}_{A}(M)$ to $\mathcal{S}_{A}(F)$. The polynomial $P(n)$ corresponding to $\lambda(n)$ can then be written in the form

$$
P(n)=\sum_{i=0}^{d+r-1}(-1)^{i} e_{i}\left(\begin{array}{c}
n+d+r-2-i \\
d+r-1-i
\end{array}\right)
$$

with integer coefficients $e_{i}$. The Buchsbaum-Rim multiplicity of $M$ in $F$, denoted by $e(F / M)$, is now defined to be the coefficient $e_{0}$.

Buchsbaum and Rim also introduced in their article the notion of a parameter module (matrix), which generalizes the notion of a parameter ideal (system of parameters). The module $N$ in $F$ is said to be a parameter module in $F$ if the following three conditions are satisfied: (i) $F / N$ has finite length, (ii) $N \subseteq \mathfrak{m} F$,

Received by the editors August 17, 2008, and, in revised form, July 14, 2009.

2000 Mathematics Subject Classification. Primary 13H15; Secondary 13D25.

Key words and phrases. Buchsbaum-Rim multiplicity, parameter module, Euler-Poincaré characteristic, generalized Koszul complex.

(C)2009 American Mathematical Society Reverts to public domain 28 years from publication 
and (iii) $\mu_{A}(N)=d+r-1$, where $\mu_{A}(N)$ is the minimal number of generators of $N$.

Buchsbaum and Rim utilized in their study the relationship between the Buchsbaum-Rim multiplicity and the Euler-Poincaré characteristic of a certain complex and proved the following:

Theorem 1.1 (Buchsbaum-Rim [5, Corollary 4.5]). Let (A, m) be a Noetherian local ring of dimension $d>0$. Then the following statements are equivalent:

(1) $A$ is a Cohen-Macaulay local ring.

(2) For any rank $r>0$, the equality $\ell_{A}(F / N)=e(F / N)$ holds true for every parameter module $N$ in $F=A^{r}$.

Then it is natural to ask the following:

Question 1.2. (1) Does the inequality $\ell_{A}(F / N) \geq e(F / N)$ hold true for any parameter module $N$ in $F$ ?

(2) Does the equality $\ell_{A}(F / N)=e(F / N)$ for some parameter module $N$ in $F$ imply that the $\operatorname{ring} A$ is Cohen-Macaulay?

The purpose of this article is to give a complete answer to Question 1.2. Our results can be summarized as follows:

Theorem 1.3. Let $(A, \mathfrak{m})$ be a Noetherian local ring of dimension $d>0$.

(1) For any rank $r>0$, the two inequalities

$$
\ell_{A}(F / N) \geq e(F / N) \text { and } \ell_{A}(A / I(N)) \geq e(F / N)
$$

always hold true for every parameter module $N$ in $F=A^{r}$, where $I(N)$ is the 0 -th Fitting ideal of $F / N$.

(2) The following statements are equivalent:

(i) $A$ is a Cohen-Macaulay local ring.

(ii) For some rank $r>0$, there exists a parameter module $N$ in $F=A^{r}$ such that the equality $\ell_{A}(F / N)=e(F / N)$ holds true.

(iii) For some rank $r>0$, there exists a parameter module $N$ in $F=A^{r}$ such that the equality $\ell_{A}(A / I(N))=e(F / N)$ holds true.

When this is the case, the equality $\ell_{A}(F / N)=\ell_{A}(A / I(N))=e(F / N)$ holds true for all parameter modules $N$ in $F=A^{r}$ of any rank $r>0$.

Note that the equality $\ell_{A}(F / N)=\ell_{A}(A / I(N))$ is known by [1, 2.10].

The proof of our Theorem 1.3 will be completed in section 4 . Section 2 is of a preliminary character. In that section we will recall the definition and some basic facts about the generalized Koszul complex. In order to prove Theorem 1.3, we will investigate in section 3 the higher Euler-Poincaré characteristics of the generalized Koszul complex and show that they are non-negative. Finally, in section 4, we will obtain Theorem 1.3 as a corollary of a more general result (Theorem 4.1).

\section{Preliminaries}

In this section we will recall the definition and some basic facts about the generalized Koszul complex introduced in [3, 8] (for more details, see also [7, Appen$\operatorname{dix}$ A2.6]).

Let $A$ be a commutative Noetherian ring, and let $n \geq r>0$ be integers. Let $\mathfrak{a}=\left(a_{i j}\right)$ be an $r \times n$ matrix over $A$, and let $I_{r}(\mathfrak{a})$ denote the ideal generated by 
the maximal minors of $\mathfrak{a}$. Let $F$ and $G$ be free modules with bases $\left\{f_{1}, \ldots, f_{r}\right\}$ and $\left\{e_{1}, \ldots, e_{n}\right\}$, respectively. Let $S$ be the symmetric algebra of $F$, and let $S_{\ell}$ be the $\ell$-th symmetric power of $F$. Let $\wedge$ be the exterior algebra of $G$, and let $\wedge^{\ell}$ be the $\ell$-th exterior power of $G$. Associated with the $i$-th row $\left[a_{i 1} \cdots a_{i n}\right]$ of $\mathfrak{a}$, there is a differentiation homomorphism $\delta_{i}: \wedge \rightarrow \wedge$ given by

$$
\delta_{i}\left(f_{j_{1}} \wedge \cdots \wedge f_{j_{p}}\right)=\sum_{k=1}^{p}(-1)^{k-1} a_{i j_{k}} f_{j_{1}} \wedge \cdots \wedge \widehat{f_{j_{k}}} \wedge \cdots \wedge f_{j_{p}} .
$$

Let $f_{i}: S \rightarrow S$ and $f_{i}^{-1}: S \rightarrow S$ denote the multiplication and division maps by $f_{i}$, respectively, i.e.,

$$
f_{i}^{-1}\left(f_{1}^{\mu_{1}} \cdots f_{i}^{\mu_{i}} \cdots f_{r}^{\mu_{r}}\right)= \begin{cases}f_{1}^{\mu_{1}} \cdots f_{i}^{\mu_{i}-1} \cdots f_{r}^{\mu_{r}} & \left(\mu_{i}>0\right) \\ 0 & \left(\mu_{i}=0\right) .\end{cases}
$$

Then the generalized Koszul complex $K_{\bullet}(\mathfrak{a} ; t)$ associated to a matrix $\mathfrak{a}$ and an integer $t$ is the complex

$$
K_{\bullet}(\mathfrak{a} ; t): \cdots \rightarrow K_{p+1}(\mathfrak{a} ; t) \stackrel{d_{p+1}}{\rightarrow} K_{p}(\mathfrak{a} ; t) \stackrel{d_{p}}{\rightarrow} K_{p-1}(\mathfrak{a} ; t) \rightarrow \cdots
$$

defined by

$$
K_{p}(\mathfrak{a} ; t)= \begin{cases}\wedge^{r+p-1} \otimes_{A} S_{p-t-1} & (p \geq t+1) \\ \wedge^{p} \otimes_{A} S_{t-p} & (p \leq t)\end{cases}
$$

and

$$
d_{p+1}= \begin{cases}\sum_{j=1}^{r} \delta_{j} \otimes f_{j}^{-1} & (p>t) \\ \delta_{r} \circ \cdots \circ \delta_{1} \otimes 1 & (p=t) \\ \sum_{j=1}^{r} \delta_{j} \otimes f_{j} & (p<t) .\end{cases}
$$

The generalized Koszul complex $K_{\bullet}(\mathfrak{a} ; t)$ is a free complex of $A$-modules. We note that it is of length $n-r+1$ when $-1 \leq t \leq n-r+1$. Also recall that $K_{\bullet}(\mathfrak{a} ; t)$ coincides with the ordinary Koszul complex for any $t$ in the case $r=1$, whereas $K_{\bullet}(\mathfrak{a} ; 0)$ is the Eagon-Northcott complex and $K_{\bullet}(\mathfrak{a} ; 1)$ is the Buchsbaum-Rim complex. Moreover, the generalized Koszul complex has the following important properties (see [8, 10] and [7, Appendix A2.6]):

Proposition 2.1. Let $\mathfrak{a}$ be an $r \times n$ matrix over $A$ with $n \geq r>0$. Then

(1) [8, Theorem 1] For any $t, p \in \mathbb{Z}, I_{r}(\mathfrak{a}) H_{p}\left(K_{\bullet}(\mathfrak{a} ; t)\right)=(0)$.

(2) [7, Theorem A2.10] If the grade of $I_{r}(\mathfrak{a})$ is at least $n-r+1$, then $K_{\bullet}(\mathfrak{a} ; t)$ is acyclic for all $-1 \leq t \leq n-r+1$. Furthermore, if $\mathfrak{a}$ is a generic matrix, then $K_{\bullet}(\mathfrak{a} ; t)$ is acyclic for all $t \geq-1$.

\section{Higher Euler-Poincaré characteristics}

In this section we will investigate higher Euler-Poincaré characteristics of a generalized Koszul complex.

Throughout this section, let $(A, \mathfrak{m})$ be a Noetherian local ring of dimension $d>0$. Let $F=A^{r}$ be a free module of rank $r>0$ with a basis $\left\{f_{1}, \ldots, f_{r}\right\}$. Let $M$ be a submodule of $F$ generated by $c_{1}, c_{2}, \ldots, c_{n}$, where $n=\mu_{A}(M)$ is the minimal number of generators of $M$. Writing $c_{j}=c_{1 j} f_{1}+\cdots+c_{r j} f_{r}$ for some $c_{i j} \in A$, we have an $r \times n$ matrix $\left(c_{i j}\right)$ associated to $M$. We call this matrix the matrix of $M$ and denote it by $\widetilde{M}$. Let $I(M)=\operatorname{Fitt}_{0}(F / M)$ be the 0-th Fitting ideal of $F / M$. We assume that $F / M$ has finite length and $M \subseteq \mathfrak{m} F$. Then $I(M)$ is an m-primary ideal, because $\sqrt{I(M)}=\sqrt{\operatorname{Ann}_{A}(F / M)}$. Hence each homology 
module $H_{p}\left(K_{\bullet}(\widetilde{M} ; t)\right)$ has finite length by Proposition 2.1(1). So the Euler-Poincaré characteristics of $K_{\bullet}(\widetilde{M} ; t)$ can be defined as follows:

Definition 3.1. For any integer $q \geq 0$, we set

$$
\chi_{q}\left(K_{\bullet}(\widetilde{M} ; t)\right):=\sum_{p \geq q}(-1)^{p-q} \ell_{A}\left(H_{p}\left(K_{\bullet}(\widetilde{M} ; t)\right)\right)
$$

and call it the $q$-th partial Euler-Poincaré characteristic of $K_{\bullet}(\widetilde{M} ; t)$. When $q=$

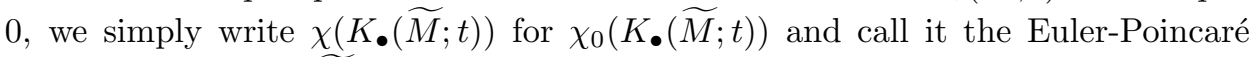
characteristic of $K_{\bullet}(\widetilde{M} ; t)$.

Buchsbaum and Rim studied in 5] the Euler-Poincaré characteristic of the Buchsbaum-Rim complex in analogy with the Euler-Poincaré characteristic of the ordinary Koszul complex in the case of usual multiplicities. In 1985 Kirby investigated in [9] Euler-Poincaré characteristics of the complex $K_{\bullet}(\widetilde{M} ; t)$ for all $t$ and proved the following:

Theorem 3.2 (Buchsbaum-Rim, Kirby). For any integer $t \in \mathbb{Z}$, we have

$$
\chi(K \bullet(\widetilde{M} ; t))=\left\{\begin{array}{cc}
e(F / M) & (n=d+r-1), \\
0 & (n>d+r-1),
\end{array}\right.
$$

where $n=\mu_{A}(M)$ is the minimal number of generators of $M$. In particular, $\chi\left(K_{\bullet}(\widetilde{M} ; t)\right) \geq 0$ for all $t \in \mathbb{Z}$.

The last statement holds for the higher Euler-Poincaré characteristics, too:

Theorem 3.3. For any $q \geq 0$ and any $t \geq-1$, we have

$$
\chi_{q}\left(K_{\bullet}(\widetilde{M} ; t)\right) \geq 0 .
$$

Proof. We use ideas from [6]. Let $\widetilde{M}=\left(c_{i j}\right) \in \operatorname{Mat}_{r \times n}(A)$ be the matrix of $M$, and let $X=\left(X_{i j}\right)$ be the generic matrix of the same size $r \times n$. Let $A[X]=A\left[X_{i j}\right.$ $1 \leq i \leq r, 1 \leq j \leq n]$ be a polynomial $\operatorname{ring}$ over $A$, and let $B=A[X]_{(\mathfrak{m}, X)}$. We will consider the ring $A$ as a $B$-algebra via the substitution homomorphism $\phi: B \rightarrow A ; X_{i j} \mapsto c_{i j}$. Let

$$
\mathfrak{b}=\operatorname{Ker} \phi=\left(X_{i j}-c_{i j} \mid 1 \leq i \leq r, 1 \leq j \leq n\right) B .
$$

We note here that $K_{\bullet}(X ; t) \otimes_{B} A \cong K_{\bullet}(\widetilde{M} ; t)$, because the generalized Koszul complex is compatible with the base change. Let $C_{t}(X):=H_{0}\left(K_{\bullet}(X ; t)\right)$. By Proposition 2.1 (2), the complex $K_{\bullet}(X ; t)$ is a $B$-free resolution of the $B$-module $C_{t}(X)$ for any $t \geq-1$. By tensoring with $A$ and taking the homology, we have that

$$
\begin{aligned}
H_{p}\left(K_{\bullet}(\widetilde{M} ; t)\right) & \cong H_{p}\left(K_{\bullet}(X ; t) \otimes_{B} A\right) \\
& \cong \operatorname{Tor}_{p}^{B}\left(C_{t}(X), A\right)
\end{aligned}
$$

for all $p \geq 0$. On the other hand, since the ideal $\mathfrak{b}$ in $B$ is generated by a regular sequence of length $r n$, the ordinary Koszul complex $K_{\bullet}(\mathfrak{b})$ associated to the sequence $\widetilde{\mathfrak{b}}$ is a $B$-free resolution of $A$. Hence, by tensoring with $C_{t}(X)$, we can compute the Tor as follows:

$$
\operatorname{Tor}_{p}^{B}\left(C_{t}(X), A\right) \cong H_{p}\left(K_{\bullet}(\mathfrak{b}) \otimes_{B} C_{t}(X)\right) .
$$

Therefore, for any $p \geq 0$, we have

$$
H_{p}\left(K_{\bullet}(\widetilde{M} ; t)\right) \cong H_{p}\left(K_{\bullet}(\mathfrak{b}) \otimes_{B} C_{t}(X)\right)
$$


It follows that for any $t \geq-1$ and any $q \geq 0$ we have the equality

$$
\chi_{q}\left(K_{\bullet}(\widetilde{M} ; t)\right)=\chi_{q}\left(K_{\bullet}(\mathfrak{b}) \otimes_{B} C_{t}(X)\right) .
$$

Here the right hand side is non-negative by Serre's Theorem ([12, Ch. IV, Appen$\operatorname{dix} \mathrm{II}])$. Therefore $\chi_{q}\left(K_{\bullet}(\widetilde{M} ; t)\right) \geq 0$.

\section{Proof of Theorem 1.3}

Theorem 1.3 will be a consequence of the following more general result:

Theorem 4.1. Let $(A, \mathfrak{m})$ be a Noetherian local ring of dimension $d>0$.

(1) For any rank $r>0$, the inequality $\ell_{A}\left(H_{0}\left(K_{\bullet}(\tilde{N} ; t)\right)\right) \geq e(F / N)$ holds true for any integer $t \geq-1$ and any parameter module $N$ in $F=A^{r}$.

(2) The following statements are equivalent:

(i) $A$ is a Cohen-Macaulay local ring.

(ii) For some rank $r>0$, there exists an integer $-1 \leq t \leq d$ and a parameter module $N$ in $F=A^{r}$ such that the equality $\ell_{A}\left(H_{0}(K \bullet(\tilde{N} ; t))\right)=$ $e(F / N)$ holds true.

When this is the case, the equality $\ell_{A}\left(H_{0}\left(K_{\bullet}(\tilde{N} ; t)\right)\right)=e(F / N)$ holds true for any integer $-1 \leq t \leq d$ and any parameter module $N$ in $F=A^{r}$ of any rank $r>0$.

Proof. (1): Let $N$ be a parameter module in $F=A^{r}$, and let $t \geq-1$. By Theorem 3.2 we obtain that

$$
\begin{aligned}
e(F / N) & =\chi\left(K_{\bullet}(\widetilde{N} ; t)\right) \\
& =\ell_{A}\left(H_{0}\left(K_{\bullet}(\widetilde{N} ; t)\right)\right)-\chi_{1}\left(K_{\bullet}(\widetilde{N} ; t)\right) .
\end{aligned}
$$

Since $\chi_{1}\left(K_{\bullet}(\tilde{N} ; t)\right) \geq 0$ by Theorem 3.3 , the desired inequality follows.

(2): Assume that $A$ is Cohen-Macaulay. Let $N$ be any parameter module in $F=A^{r}$ of any rank $r>0$. Let $n=\mu_{A}(N)=d+r-1$. Then grade $I(N)=$ ht $I(N)=d=n-r+1$. Hence, by Proposition 2.1 (2), $K_{\bullet}(\tilde{N} ; t)$ is acyclic for all $-1 \leq t \leq n-r+1=d$. Therefore, by Theorem 3.2. we have $e(F / N)=$ $\chi\left(K_{\bullet}(\tilde{N} ; t)\right)=\ell_{A}\left(H_{0}\left(K_{\bullet}(\tilde{N} ; t)\right)\right)$. This proves the implication (i) $\Rightarrow$ (ii) and also the last assertion.

It remains to show the implication (ii) $\Rightarrow$ (i). Assume that there exist integers $r>$ $0,-1 \leq t \leq d$, and a parameter module $N$ in $F=A^{r}$ such that $\ell_{A}\left(H_{0}\left(K_{\bullet}(\widetilde{N} ; t)\right)\right)=$ $e(F / N)$. Arguing as in the proof of Theorem 3.3 and using the same notation, we get

$$
\begin{aligned}
\chi_{1}\left(K_{\bullet}(\mathfrak{b}) \otimes_{B} C_{t}(X)\right) & =\chi_{1}\left(K_{\bullet}(\tilde{N} ; t)\right) \\
& =\ell_{A}\left(H_{0}(K \bullet(\widetilde{N} ; t))\right)-e(F / N) \\
& =0 .
\end{aligned}
$$

We observe here that $\sqrt{\operatorname{Ann}_{B} C_{t}(X)}=\sqrt{I_{r}(X)}$ (see [11, Lemma 2.7]). Thus $\operatorname{dim}_{B} C_{t}(X)=\operatorname{dim} B / I_{r}(X)=d+(n+1)(r-1)=r n$ (see [2, (5.12), Corollary]). Therefore $\mathfrak{b}$ is a parameter ideal of $C_{t}(X)$. Hence we have the equality

$$
\ell_{B}\left(C_{t}(X) / \mathfrak{b} C_{t}(X)\right)-e\left(\mathfrak{b} ; C_{t}(X)\right)=\chi_{1}\left(K_{\bullet}(\mathfrak{b}) \otimes_{B} C_{t}(X)\right)=0,
$$

where $e\left(\mathfrak{b} ; C_{t}(X)\right)$ is the multiplicity of the module $C_{t}(X)$ with respect to an ideal $\mathfrak{b}$. Since $\ell_{B}\left(C_{t}(X) / \mathfrak{b} C_{t}(X)\right)=e\left(\mathfrak{b} ; C_{t}(X)\right)$, this implies that $C_{t}(X)$ is a CohenMacaulay $B$-module. On the other hand, $\operatorname{pd}_{B} C_{t}(X)=d$, because the complex 
$K_{\bullet}(X ; t)$ is a minimal $B$-free resolution of $C_{t}(X)$ of length $n-r+1=d$. Hence, by the Auslander-Buchsbaum formula, we have

$$
\begin{aligned}
d+r n & =\operatorname{pd}_{B} C_{t}(X)+\operatorname{depth}_{B} C_{t}(X) \\
& =\operatorname{depth} B \\
& \leq \operatorname{dim} B \\
& =d+r n .
\end{aligned}
$$

Thus depth $B=\operatorname{dim} B$ so that $B$ is Cohen-Macaulay. Therefore $A$ is also a CohenMacaulay local ring.

Taking $t=0,1$ in Theorem 4.1 now readily gives Theorem 1.3

We want to close this article with a question. For that, let us first recall the notion of a Buchsbaum local ring, which was introduced by Stückrad and Vogel (for more details on Buchsbaum rings, we refer the reader to [13]). Let $A$ be a Noetherian local ring. Then $A$ is said to be a Buchsbaum ring if the difference

$$
\ell_{A}(A / Q)-e(A / Q)
$$

between the colength and multiplicity of a parameter ideal $Q$ in $A$ is independent of the choice of $Q$. This difference, which is an invariant of a Buchsbaum ring $A$, is denoted by $I(A)$. The ring $A$ is Cohen-Macaulay if and only if it is Buchsbaum and $I(A)=0$. In this sense, the notion of a Buchsbaum ring is a natural generalization of that of a Cohen-Macaulay ring. In Theorem 1.3, the inequality $\ell_{A}(F / N) \geq e(F / N)$, for any parameter module $N$ in $F$, is an analogue of the well-known inequality $\ell_{A}(A / Q) \geq e(A / Q)$ for any parameter ideal $Q$ in $A$. Also, the characterization of the Cohen-Macaulay property of $A$ based on the equality $\ell_{A}(F / N)=e(F / N)$ generalizes the usual one using parameter ideals. With these remarks in mind, it is natural to ask the following question:

Question 4.2. Let $F$ be a fixed free module of rank $r>0$. Is it then true that the difference

$$
\ell_{A}(F / N)-e(F / N)
$$

between the colength and multiplicity of a parameter module $N$ in $F$ is independent of the choice of $N$ if the ring $A$ is Buchsbaum?

\section{REFERENCES}

[1] W. Bruns and U. Vetter, Length formulas for the local cohomology of exterior powers, Math. Z. 191 (1986), 145-158. MR812608 (87c:13016)

[2] W. Bruns and U. Vetter, Determinantal Rings, Lecture Notes in Math., 1327, Springer-Verlag, Berlin-Heidelberg, 1988. MR953963 (89i:13001)

[3] D. A. Buchsbaum and D. Eisenbud, Generic free resolutions and a family of generically perfect ideals, Adv. in Math. 18 (1975), 245-301. MR0396528 (53:391)

[4] D. A. Buchsbaum and D. S. Rim, A generalized Koszul complex, Bull. Amer. Math. Soc. 69 (1963), 382-385. MR0148720(26:6226)

[5] D. A. Buchsbaum and D. S. Rim, A generalized Koszul complex. II. Depth and multiplicity, Trans. Amer. Math. Soc. 111 (1964), 197-224. MR0159860 (28:3076)

[6] D. A. Buchsbaum and D. S. Rim, A generalized Koszul complex. III. A remark on generic acyclicity, Proc. Amer. Math. Soc. 16 (1965), 555-558. MR0177020 (31:1285)

[7] D. Eisenbud, Commutative algebra. With a view toward algebraic geometry, Graduate Texts in Mathematics, 150, Springer-Verlag, New York, 1995. MR1322960(97a:13001)

[8] D. Kirby, A sequence of complexes associated with a matrix, J. London Math. Soc. 7 (1974), 523-530. MR0337939(49:2708) 
[9] D. Kirby, On the Buchsbaum-Rim multiplicity associated with a matrix, J. London Math. Soc. (2) 32 (1985), no. 1, 57-61. MR813385 (87d:13025)

[10] D. Kirby, Generalized Koszul complexes and the extension functor, Comm. Algebra 18 (1990), no. 4, 1229-1244. MR.1059948 (91e:13015)

[11] A. G. Rodicio, On the rigidity of the generalized Koszul complexes with applications to Hochschild homology, J. Algebra 167 (1994), no. 2, 343-347. MR.1283291 (95e:13011)

[12] J-P. Serre, Local Algebra (translated from the French by CheeWhye Chin), Springer Monographs in Mathematics, Springer-Verlag, Berlin-Heidelberg, 2000. MR 1771925(2001b:13001)

[13] J. Stückrad and W. Vogel, Buchsbaum Rings and Applications, Springer-Verlag, BerlinHeidelberg-New York-London-Paris-Tokyo, 1986. MR881220 (88h:13011a)

Department of Mathematics, School of Science and Technology, Meiji University, 1-1-1 Higashimita, Tama-Ku, KaWasaki 214-8571, Japan

E-mail address: hayasaka@isc.meiji.ac.jp

Department of Mathematics and Statistics, University of Tampere, 33014 Tampereen YLIOPISTO, FinLAND

E-mail address: Eero.Hyry@uta.fi 